\title{
Differential activation patterns of occipital and prefrontal cortices during motion processing: Evidence from normal and schizophrenic brains
}

\author{
Yue CHeN \\ Harvard Medical School, Belmont, Massachusetts \\ Emily D. Grossman \\ University of California, Irvine, California \\ L. Cinnamon Bidwell \\ University of Colorado, Boulder, Colorado
}

\author{
Deborah Yurgelun-Todd, Staci A. Gruber, and Deborah L. Levy \\ Harvard Medical School, Belmont, Massachusetts \\ Ken NAKayama \\ Harvard University, Cambridge, Massachusetts
}

AND

Philip S. Holzman

Harvard Medical School, Belmont, Massachusetts

and Harvard University, Cambridge, Massachusetts

\begin{abstract}
Visual motion perception is normally mediated by neural processing in the posterior cortex. Focal damage to the middle temporal area (MT), a posterior extrastriate region, induces motion perception impairment. It is unclear, however, how more broadly distributed cortical dysfunction affects this visual behavior and its neural substrates. Schizophrenia manifests itself in a variety of behavioral and perceptual abnormalities that have proved difficult to understand through a dysfunction of any single brain system. One of these perceptual abnormalities involves impaired motion perception. Motion processing provides an opportunity to clarify the roles of multiple cortical networks in both healthy and schizophrenic brains. Using fMRI, we measured cortical activation while participants performed two visual motion tasks (direction discrimination and speed discrimination) and one nonmotion task (contrast discrimination). Normal controls showed robust cortical activation (BOLD signal changes) in MT during the direction and speed discrimination tasks, documenting primary processing of sensory input in this posterior region. In patients with schizophrenia, cortical activation was significantly reduced in MT and significantly increased in the inferior convexity of the prefrontal cortex, an area that is normally involved in higher level cognitive processing. This shift in cortical responses from posterior to prefrontal regions suggests that motion perception in schizophrenia is associated with both deficient sensory processing and compensatory cognitive processing. Furthermore, this result provides evidence that in the context of broadly distributed cortical dysfunction, the usual functional specificity of the cortex becomes modified, even across the domains of sensory and cognitive processing.
\end{abstract}

Functional specificity is a fundamental property of brain-behavior relationships (Brodmann, 1909; Edelman, 2003; Goldman-Rakic, 1988; Van Essen \& Maunsell, 1983). The posterior cortex, for instance, mediates primarily sensory processes, whereas the anterior cortex mediates primarily cognitive processes. The cortex is also plastic, in that it can adjust its functionality to adapt to changes in its own system and in the external world. For example, focal damage to motion-sensitive areas (such as the middle temporal area, or MT) induces an acute deficit in motion perception (Newsome \& Pare, 1988), an example of functional specificity between the posterior cortex and sensory responses. This induced perceptual deficit can recover over time, when other parts of the same cortical area adjacent to the focal damage are recruited (Plant \& Nakayama, 1993; Wurtz, Yamasaki, Duffy, \& Roy, 1990). 
Research on visual motion processing has focused primarily on the occipital cortex, because motion perception has generally been considered a task mediated in regions such as MT. However, several lines of evidence have recently made it clear that the prefrontal cortex (PFC) also participates in neural responses to motion stimuli. First, neural responses in the PFC are not only sensitive to the presence of moving targets, but are also modulated by the specifics of motion task demands (Zaksas \& Pasternak, 2006). Second, there is a reciprocal relationship between motion signal strength and neural activity in anterior and posterior regions: Anterior cortical activity decreases, and posterior cortical activity increases, as a function of the strength of motion stimuli (Rees, Friston, \& Koch, 2000). The PFC is likely part of a default-mode network, showing deactivation during a low-load task or during resting conditions (Greicius \& Menon, 2004). It remains unknown, however, whether these functional roles of the PFC change when sensory processing of motion information in the occipital cortex is deficient.

A more general question concerns the relationship between dysfunction involving more broadly distributed cortical networks, which are strongly implicated in schizophrenia (Andreasen, 1999; Coyle, 1996), and specific behaviors (e.g., visual motion perception).

Unlike neurological disorders in which localized cortical damage produces impairments in specific behaviors, schizophrenia shows few signs of consistent gross histopathological changes in any single brain area (Benes, 2000). In contrast, schizophrenia manifests itself in a variety of striking behavioral abnormalities, such as hallucinations, delusions, blunted emotional expression, disorganized thinking, and difficulty maintaining attention (Bleuler, 1950; Kraepelin, 1919). The coexistence of prominent behavioral and subtle brain structural changes challenges the notion that schizophrenia can be understood solely on the basis of localized brain dysfunction. Rather, the anatomical and behavioral findings are consistent with the presence of more distributed brain disorganization.

Schizophrenia provides an opportunity to examine how compromise involving broadly distributed cortical networks, rather than a single cortical system, affects behavioral performance. In order to tap into the functional organization of schizophrenic brains, it is essential for one to select behaviors that are dysfunctional in schizophrenia and whose underlying neural mechanisms involve multiple cortical systems. Smooth pursuit eye movements require the involvement of a network of brain areas, in- cluding occipital, parietal, and frontal cortices (Keller \& Heinen, 1991; Newsome \& Pare, 1988; Wurtz et al., 1990) and are significantly impaired in patients with schizophrenia and their first-degree relatives (Holzman, Proctor, \& Hughes, 1973; Levin et al., 1988; Levy, Holzman, Matthysse, \& Mendell, 1993; Sweeney et al., 1994; Thaker et al., 1998). Psychophysical studies implicate a defect in visual motion processing - normally mediated in the extrastriate cortex - in SPEM impairment in schizophrenia (Chen, Nakayama, Levy, Matthysse, \& Holzman, 1999; Kim, Wylie, Pasternak, Butler, \& Javitt, 2006; Stuve et al., 1997). Motion perception in schizophrenia is thus suitable for studying the relationship between multiple cortical systems and behavior.

In the present study, we utilized neuroimaging techniques and psychophysical methods in order to examine the functional integrity of cortical activation in sensory visual areas during motion and nonmotion discrimination tasks in patients with schizophrenia. We also explored whether cortical areas that are normally involved in cognitive processing — but not in sensory visual processing are activated during visual discrimination.

\section{METHOD}

\section{Participants}

Ten outpatients who met the Diagnostic and Statistical Manual of Mental Disorders (4th ed.) (DSM-IV; American Psychiatric Association, 1994) criteria for schizophrenia or schizoaffective disorder were included in the study. The patients were chronically ill (duration of illness: $M=15.1$ years, $S D=6.7$ ) and moderately symptomatic (BPRS: $M=27.3, S D=4.9$ ). Consensus diagnoses were made independently by experienced clinicians, based on a review of a structured clinical interview for $D S M-I V$ conducted by trained interviewers (Spitzer, Williams, Gibbon, \& First, 1994) and on an evaluation of all available hospital records. Nine patients were taking antipsychotic medication (daily chlorpromazine equivalent: $M=$ $413 \mathrm{mg}, S D=27$ ) (Woods, 2003). None of the eight normal controls met $D S M-I V$ criteria for any psychotic condition (lifetime); for any schizotypal, schizoid, or paranoid personality disorder, based on the Structured Interview for Schizotypy (Kendler, Leiberman, \& Walsh, 1989; Spitzer et al., 1994); or had a family history of psychosis. All participants were right-handed and native English speakers. They had no diagnosed organic brain disease and no history of substance abuse or dependence during the past 2 years. The groups were matched on age, gender, years of education, and estimated verbal IQ (Table 1). Written informed consent was obtained prior to testing.

\section{Visual Discrimination Tasks}

Three visual discrimination tasks were used. Two were motion tasks, on which patients with schizophrenia have previously demonstrated impairments (Chen, Nakayama, et al., 1999; Chen, Nakayama, Levy, Matthysse, \& Holzman, 2003)—direction and speed

Table 1

Demographic Characteristics of Participants

\begin{tabular}{|c|c|c|c|c|c|c|c|c|c|c|c|c|}
\hline \multirow[b]{2}{*}{ Group } & \multicolumn{2}{|c|}{ Sex } & \multicolumn{2}{|c|}{ Age } & \multicolumn{4}{|c|}{ SES (\%) } & \multicolumn{2}{|c|}{ Verbal IQ } & \multicolumn{2}{|c|}{ Education } \\
\hline & Male & Female & $M$ & $S D$ & I & II & III & IV & $M$ & $S D$ & $M$ & $S D$ \\
\hline Control & 4 & 4 & 39.5 & 12.3 & 12.5 & 75 & 12.5 & - & 103.8 & 10.3 & 16.0 & 2.8 \\
\hline Patient & 5 & 5 & 38.5 & 8.7 & 40 & 30 & 20 & 10 & 105.5 & 8.3 & 14.4 & 2.5 \\
\hline
\end{tabular}

Note-SES, social-economical status (based on Hollingshead-Redlich Two-Factor Index; Hollingshead, 1957). Age and education are given in years. 
discrimination - and the third was a nonmotion control task-contrast discrimination.

Direction discrimination. In each trial, participants indicated which of two sequentially presented random dot patterns (speed, $10 \mathrm{deg} / \mathrm{sec}$; number of dots, 400; dot lifetime, $90 \mathrm{msec}$ ) moved to the right. The random dot patterns comprised a spatially intermixed motion component - an array of dots moving coherently in one direction (left or right, randomly presented first or second in each trial) - and a noise component - another array of dots moving in random directions.

Speed discrimination. Participants indicated which of two sequentially presented gratings moved faster (spatial frequency, 0.5 cycles/deg; orientation, vertical; contrast, $20 \%$; base speed, $10 \mathrm{deg} / \mathrm{sec}$; monochromatic). The faster moving grating (e.g., $14 \mathrm{deg} / \mathrm{sec}$ ) was randomly presented first or second in each trial.

Contrast discrimination. Participants indicated which of two sequentially presented gratings had higher contrast. The gratings were identical to those used in the speed discrimination task, except that contrast, rather than speed, was varied between the two stimuli presented within each trial (e.g., $20 \%$ base contrast vs. $28 \%$ higher contrast).

In all three tasks, each stimulus was displayed within a circular window (diameter, $10^{\circ}$ ) for $300 \mathrm{msec}$, with an interstimulus interval of $500 \mathrm{msec}$. Participants were instructed to fixate on a small central target between visual discrimination tasks.

\section{Equating for Task Difficulty}

Offline testing determined each participant's perceptual threshold $(80 \%$ correct accuracy) on each of the three tasks. Functional brain images were acquired at two task difficulty levels (easy and difficult). In the difficult conditions, task difficulty level was set at twice each individual participant's offline thresholds. In the easy conditions, identical stimulus strengths were used for all participants: $50 \%$ contrast versus $20 \%$ contrast for contrast discrimination; $16 \mathrm{deg} / \mathrm{sec}$ versus $10 \mathrm{deg} / \mathrm{sec}$ for speed discrimination; and $70 \%$ motion coherence for direction discrimination.

\section{MRI Acquisition and Analysis}

Scanning was conducted on a 1.5-tesla GE Signa magnet at the Brain Imaging Center of McLean Hospital (Belmont, MA). In a 1.5-h session, we acquired high resolution anatomical images of the entire brain (spoiled-grass imaging, $0.9375 \times 0.9375 \times 2.5 \mathrm{~mm}$, 124 slices, $1.5 \mathrm{~mm}$ thick, TE $=5 \mathrm{msec}$, TR $=35 \mathrm{msec}$, flip $=45^{\circ}$ ), matched anatomical images of slice locations (19-22 axial slices, $7 \mathrm{~mm}$ thick, 1-mm gap), and six sequences of functional scans (single-shot echo-planar imaging, $3 \times 3 \mathrm{~mm}$ in-plane resolution, $\mathrm{TR}=3,000 \mathrm{msec}, \mathrm{TE}=40 \mathrm{msec}$, flip $=75^{\circ}$ ). Images were acquired with a quadrature birdcage headcoil. Participants were placed in a supine position and viewed the visual stimuli, which were backprojected on a screen located at the participant's feet through a mirror apparatus attached to the headcoil. Structural imaging data were acquired for use in the registration of the functional imaging data and were read and interpreted by a clinical neuroradiologist to ensure that participants were free of neuroradiological abnormalities.

Functional scan epochs lasted $2.5 \mathrm{~min}$ each; the initial $10 \mathrm{sec}$ were discarded prior to analysis to allow for MR stabilization. The task conditions were divided into 20 -sec blocks, alternating with 20 -sec blocks of fixation, which served as a baseline. One task condition was presented per epoch, for a total of six scan epochs (three tasks, each with two difficulty levels). The task conditions were presented in a randomized order across participants. Behavioral responses (i.e., perceptual judgments) were made with a keypress on an MRcompatible mouse device.

All image analyses were conducted using Brain Voyager (Brain Innovations, Inc.) after registering the functional images to the individual participant's anatomy and then warping into standardized stereotaxic space (Talairach \& Tournoux, 1988). Individual voxels were then corrected for linear drift in time and spatially smoothed with a 4-mm FWHM Gaussian filter.
Statistical analysis was conducted using the general linear model (GLM; Friston, Worsley, Frackowiak, Mazziotta, \& Evans, 1993), with boxcar function predictors for each task condition. Group differences were revealed by planned comparisons across populations in each task. Resulting $p$ values were corrected for multiple comparisons using a false discovery rate procedure.

Regions of interest (ROIs) were defined from the corrected results of the GLM in the easy condition of the direction discrimination task, because this task, particularly under easy conditions, has been shown to activate motion-sensitive cortical areas (Rees et al., 2000). To obtain ROIs during motion perception, we compared neural activity during blocks in which a moving target was presented and during blocks in which the target was stationary, a functional localization method developed by Tootell et al. (1995). MT+ was identified as the brain area on the ascending limb of the inferior occipital sulcus that was activated by the coherent motion stimuli in the easy direction discrimination task. The combination of functional localization with the known anatomical landmarks associated with MT+ (e.g., Huk \& Heeger, 2002) is a very reliable way of identifying this direction-selective cortical area. The ROIs were generated from the patients and controls together, as a single group.

Because an ROI analysis ignores neural events occurring outside the targeted areas, we also conducted a general linear model analysis of the whole brain. This model compared neural activity in the two groups during the three tasks. The whole-brain GLM analysis revealed a significant group difference during the motion tasks in the PFC; this difference was not predicted a priori. In addition, since there are no functional localizers that can be used to identify specific regions of $\mathrm{PFC}$ that get recruited during motion tasks, we used the whole-brain between-groups analysis to identify the PFC ROI.

Voxels within each ROI were averaged to create a single response time series for each group. In each ROI, a two-way ANOVA tested the significance of BOLD signal changes between schizophrenia patients and normal controls as main effects and the interaction between participant group and task difficulty.

\section{RESULTS}

Patients and controls performed the three visual discrimination tasks similarly within the scanner environment (Figure 1). For each of the tasks, a two-way ANOVA of group (patient, control) and task difficulty (easy, difficult) showed a significant effect of task difficulty [for contrast task, $F(1,34)=10.426, p=.003$; for velocity task, $F(1,34)=21.648, p<.0001$; for direction task, $F(1,34)=8.574, p=.006]$. Neither the group effect [contrast task, $F(1,34)=0.308, p=.583$; velocity task, $F(1,34)=1.845, p=.184$; direction task, $F(1,34)=$ $0.833, p=.368$ ] nor the interaction between group and task difficulty (contrast task, $F=0.040, p=.843$; velocity task, $F=0.139, p=.712$; direction task, $F=0.001$, $p=.981)$ was statistically significant.

In a two-stage analysis, we targeted brain areas known to be involved in motion discrimination (i.e., V1/V2, MT+) and sought to identify any regional differences in cortical activity between controls and patients in areas outside of the striate and the extrastriate cortices (i.e., using wholebrain analysis).

The direction discrimination task revealed significant group differences in neural activation patterns (Figure 2A; Table 2). A two-way ANOVA of BOLD signal changes in all four ROIs showed a significant interaction [group $X$ area: $F(1,3)=12.63, p<.001]$. Post hoc tests showed that, compared with the normal controls (light bars), pa- 


\section{Task Difficulty and Participant Performance in Scanner}

A Direction Discrimination

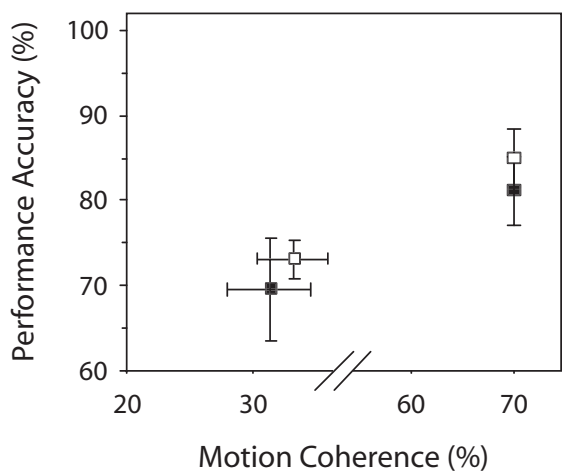

B Velocity Discrimination

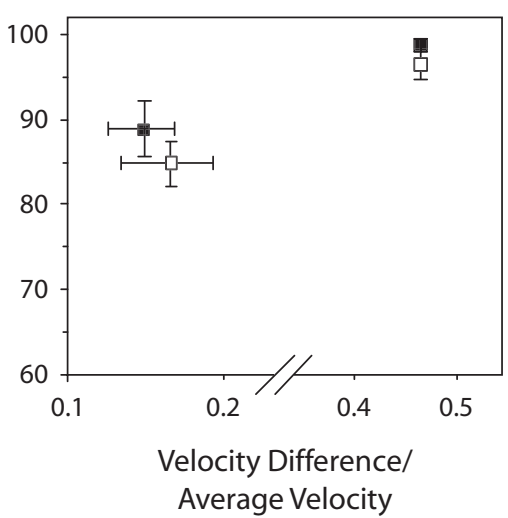

C Contrast Discrimination

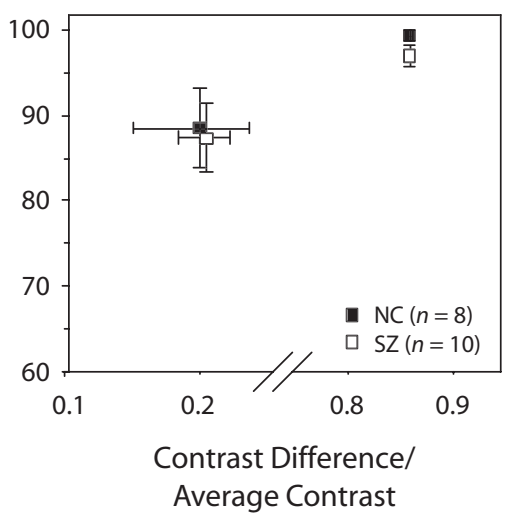

Stimulus Strength

Figure 1. Relation of stimulus strength (task difficulty) to participant performance in the scanner. In each of the three panels (A, $\mathrm{B}$, and $\mathrm{C}$, for direction, speed, and contrast discrimination, respectively), group performance is plotted on the ordinate and stimulus strength on the abscissa. The error bars indicate \pm 1 standard error. In the difficult task conditions (the left pair of data points in each panel), the stimulus strengths were twice the psychophysical thresholds determined for each participant prior to scanning. In the easy task conditions (the right pair of data points in each panel), the stimulus strengths were identical for all participants (hence, no error bars). Both participant groups performed all tasks at an accuracy level above $80 \%$ correct on the easy conditions, and at or above $70 \%$ correct on the difficult conditions.

tients with schizophrenia (dark bars) showed a significantly lower BOLD response in MT $+[F(1,34)=6.46$, $p<.02, d=0.96]$. In contrast, activation in the patients was significantly higher in the left inferior convexity of the PFC (ICPFC) [ $F(1,34)=25.31, p<.001, d=1.83$ ]. There were no significant group differences in V1/V2 $[F(1,34)=2.97, p=.09, d=0.56]$ and IFG $[F(1,34)=$ $0.33, p=.58, d=0.23]$. Group $\times$ task difficulty interactions were not significant. Figure $2 \mathrm{~B}$ shows the activation difference between the groups in $\mathrm{MT}+$ and in the left ICPFC.

A similar pattern emerged when participants performed a speed discrimination task. The average BOLD response of the schizophrenia group was significantly lower than that of the normal control group in $\mathrm{MT}+[F(1,34)=$ $7.18, p<.02, d=0.89]$. The patients showed significantly higher activation than did the controls in left ICPFC $[F(1,34)=8.73, p<.01, d=1.61]$. The two groups did not differ in V1/V2 $[F(1,34)=0.63, p=.44, d=0.35]$

Table 2

Region-of-Interest (ROI) Analysis

\begin{tabular}{|c|c|c|c|c|c|c|}
\hline \multirow[b]{3}{*}{ ROI } & \multicolumn{6}{|c|}{ Talairach Coordinates (Centroids) } \\
\hline & \multicolumn{3}{|c|}{ Left Hemisphere } & \multicolumn{3}{|c|}{ Right Hemisphere } \\
\hline & $x$ & $y$ & $z$ & $x$ & $y$ & $z$ \\
\hline V1/V2 & -14 & -82 & -8 & 10 & -84 & -8 \\
\hline $\mathrm{MT}+$ & -44 & -61 & -2 & 46 & -65 & -1 \\
\hline IFG & -32 & 18 & 3 & 31 & 19 & 7 \\
\hline ICPFC & -32 & 46 & 4 & - & - & - \\
\hline
\end{tabular}

Note-V1/V2, primary visual cortex; $\mathrm{MT}+$, middle temporal area complex; IFG, inferior frontal gyrus; ICPFC, inferior convexity of prefrontal cortex. and IFG $[F(1,34)=2.15, p=.15, d=0.54]$. The effect sizes for both results were large for the speed and direction discrimination tasks. The groups did not differ significantly in activation of the striate cortex and the IFG (Figure 3). Overall, the patients revealed a shift in activation from the posterior visual areas toward the anterior prefrontal cortex during the two motion discrimination tasks (Figure 4).

Unlike in the two motion discrimination tasks, the two groups did not differ significantly in the contrast discrimination task [for V1/V2, $F(1,34)=1.60, p=.22, d=0.43$; for MT $+, F(1,34)=1.46, p=.24, d=0.43$; for IFG, $F(1,34)=0.18, p=.67, d=0.16$; and for left ICPFC, $F(1,34)=1.00, p=.32, d=0.22]$ (see Figure 3).

The magnitude of group differences in the left ICPFC was larger in the difficult task conditions than in the easy task conditions (Figure 4), but the interaction between group (patient, control) and task difficulty (easy, difficult) was not statistically significant for any of the tasks.

\section{DISCUSSION}

We showed that motion processing in controls activates primarily striate and extrastriate areas, a finding consistent with the known functional specificity of the posterior cortex (Tootell et al., 1995). In contrast, the pattern of cortical activation subserving motion perception was altered in patients with schizophrenia. This alteration extended from visual areas (such as MT+) to prefrontal areas (such as the interior convexity of the prefrontal cortex). The results suggest that during motion processing, cortical activities in schizophrenia are altered not only in the poste- 
A
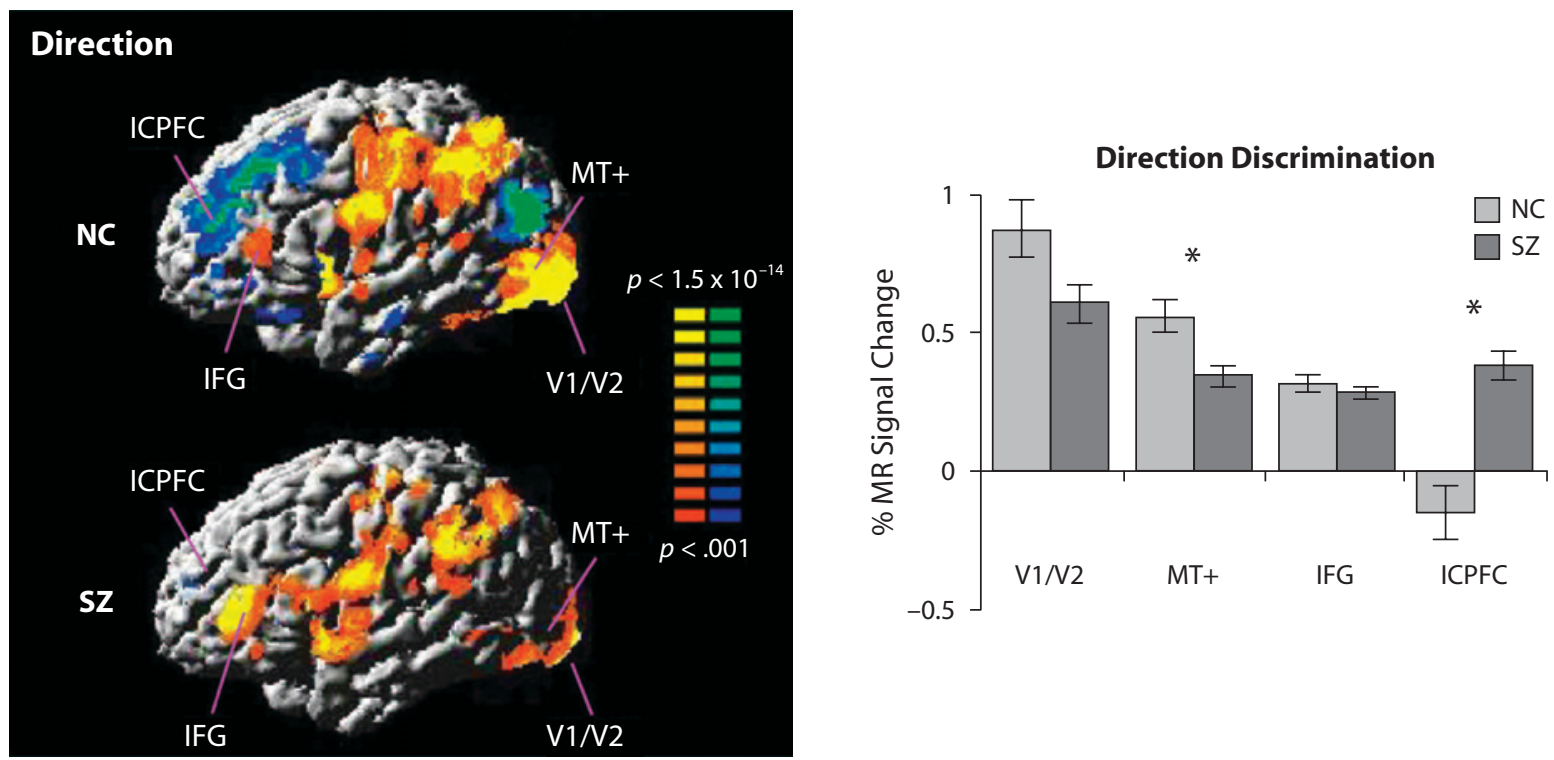

B
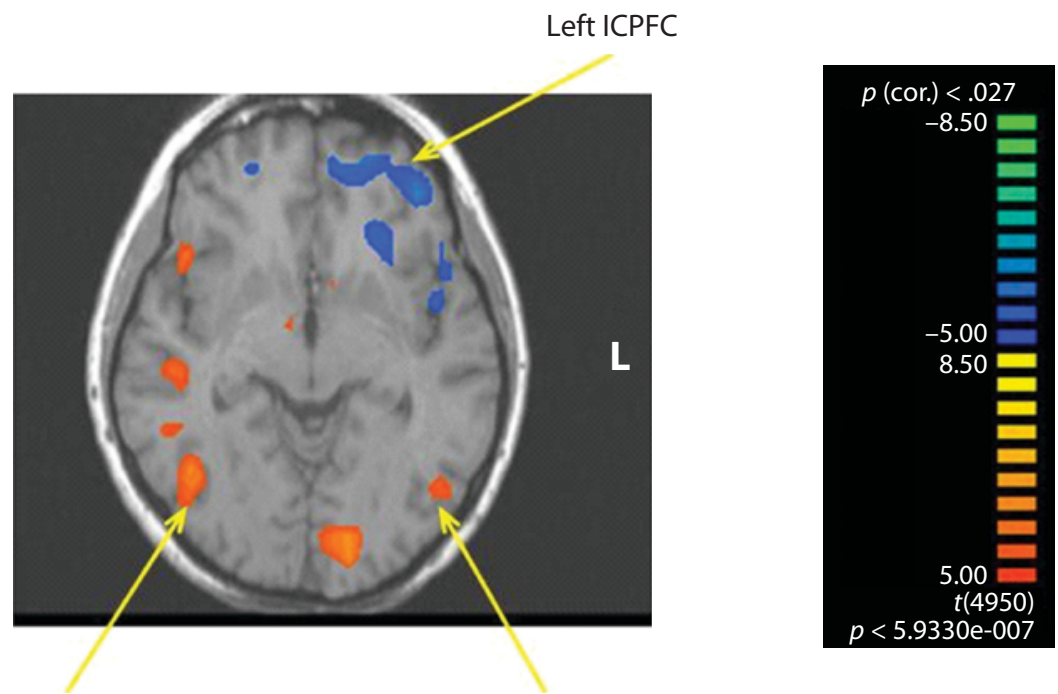

Right MT

Left MT

Figure 2. Group activation maps and histograms for the direction discrimination task. (A) Statistical maps (collapsed across task difficulty levels) of the normal controls (NC, top left) illustrate neural activity in many brain areas during this task relative to fixation, including activation (color coding: yellow/red) in posterior (e.g., occipital) regions, and deactivation (green/blue) in frontal areas. The patients with schizophrenia (SZ, bottom left) showed a different pattern. Activation in much of the occipital cortex was reduced, and activation in frontal regions was increased. The average BOLD response (expressed as the percentage of change from the fixation baseline) is shown in the histograms (right). The response represents peak and not the entire activation of the region. ICPFC data are for the left hemisphere only. The error bars indicate \pm 1 standard error. (B) Activation difference map during the direction discrimination task between the groups in MT+ and left ICPFC. With the group subtraction (NC - SZ), positive values (color coding: yellow/red) indicate a greater activation in the normal controls, whereas negative values (color coding: green/blue) indicate less activation in the normal controls. "Significant differences between the NC and SZ groups $(p<.05)$.

rior regions involved in sensory processing, but also in the anterior areas subserving cognitive processing.

\section{Behavioral Performance}

The group differences in patterns of functional activation during the two motion tasks are not likely to reflect the patients' failure to engage in the visual tasks. Both the patients and the controls were able to perform well within the scanner. In order to achieve equivalent levels of online performance in the difficult condition, we tailored each individual's online stimulus strength to that person's offline perceptual threshold for $80 \%$ accuracy. The greater 


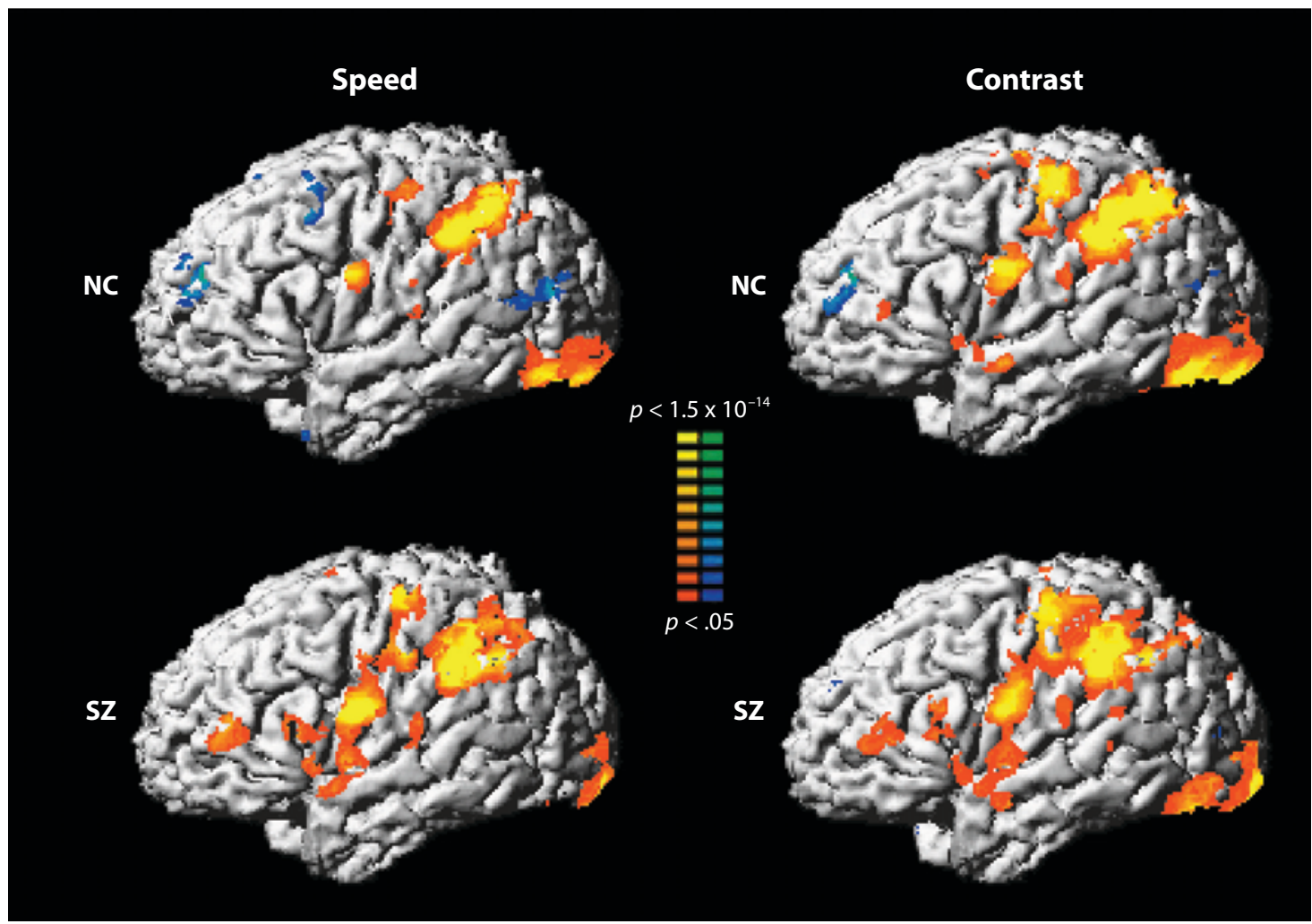

Speed Discrimination

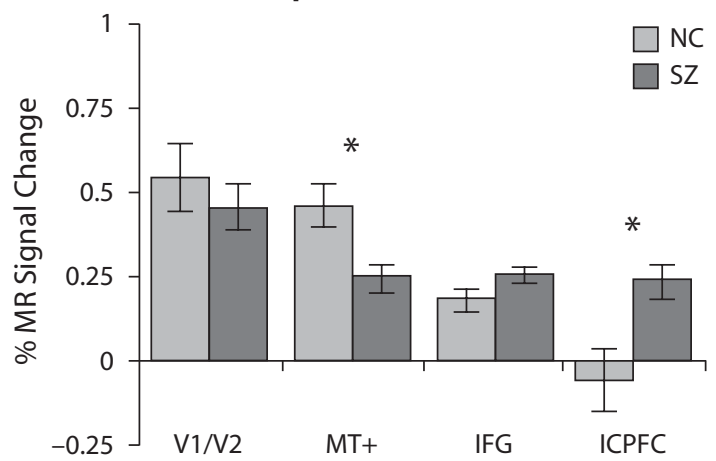

Contrast Discrimination

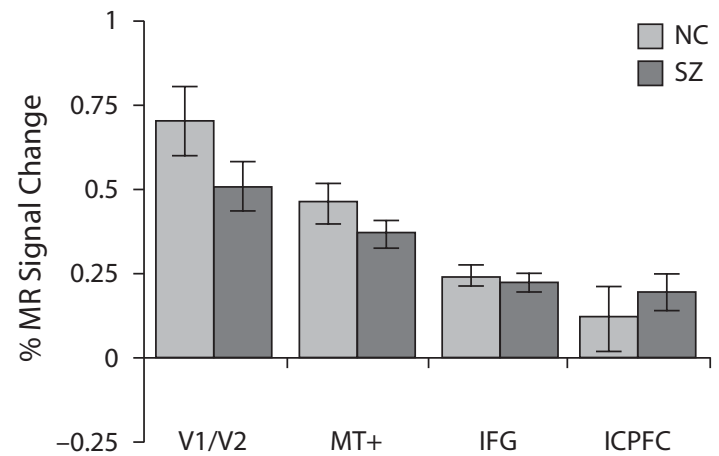

Figure 3. Group activation maps and activation histograms for the speed (left) and contrast (right) discrimination tasks. See the legend for Figure 2 for an explanation of the color coding and histogram.

stimulus strength required by the patients is consistent with previous reports of impaired speed and direction discrimination and of other visual processing deficits in schizophrenia. Had we used a common "difficult" task condition, rather than individual perceptual thresholds tied to a common accuracy criterion, the groups would likely have differed in performance accuracy, and the interpretation of group differences in functional activation would have been confounded by this difference in behavioral performance. The standardized stimulus strengths used in the easy conditions were much higher than the individual thresholds, resulting in better than $80 \%$ accuracy in both groups. All three visual discrimination tasks required comparisons of sequentially presented stimuli, but the time interval between the stimuli was brief $(500 \mathrm{msec})$ and was within the range in which patients with schizophrenia do not show working memory deficits (Park \& Holzman, 1992). Despite similar task performance in the easy and difficult conditions, cortical activation in the patients was significantly lower in extrastriate MT + and higher in left ICPFC during direction and speed discrimination. Thus, the group differences in pattern of functional activation cannot be explained as secondary effects of differences in performance.

It is noteworthy that perceptual performance was not significantly correlated with PFC or MT activity in ei- 
Group Differences in BOLD Signals

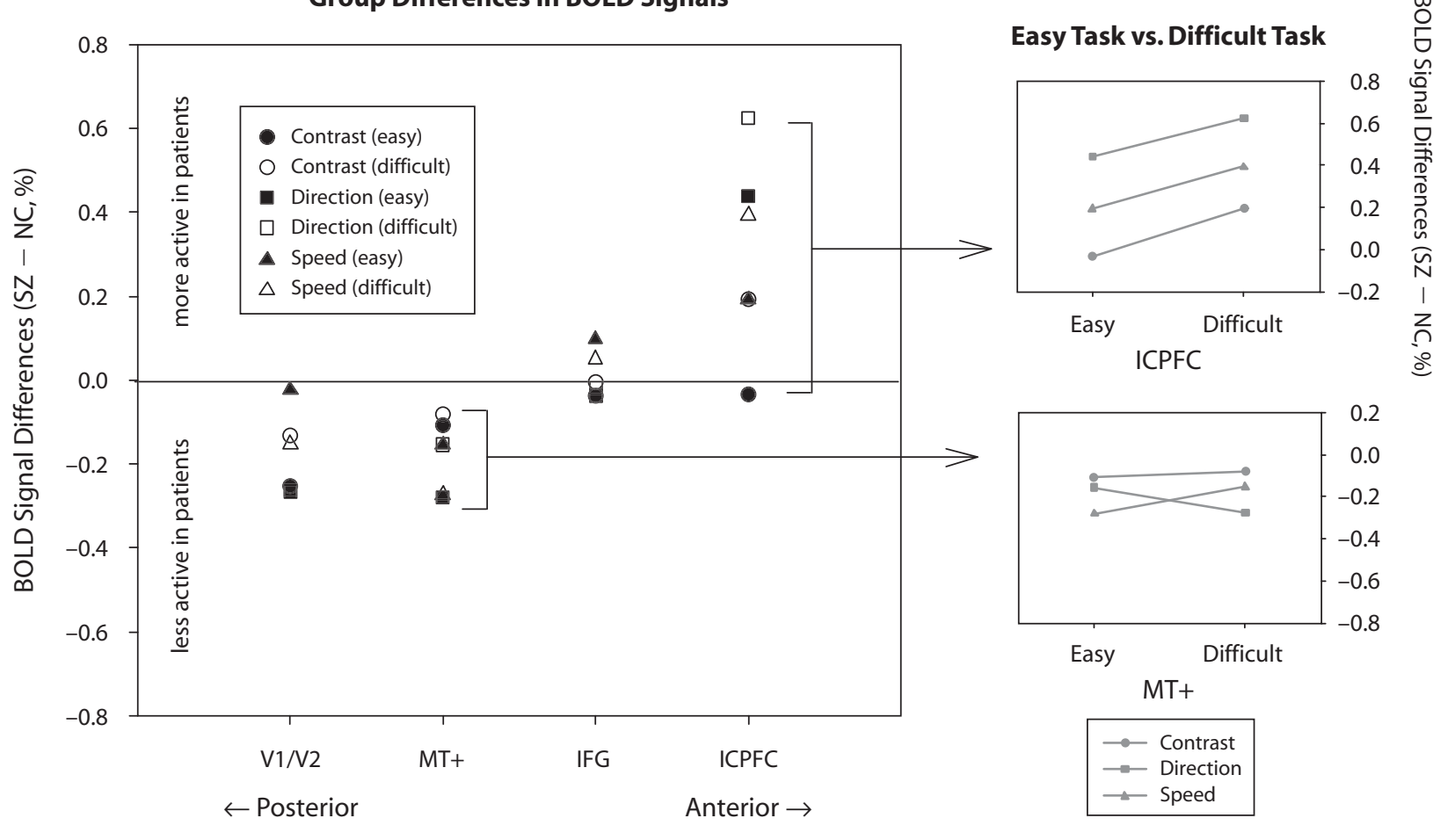

Figure 4. Summary of BOLD signal changes during the three visual discrimination tasks. Group differences (SZ - NC) in BOLD signal changes are plotted on the ordinate, and ROIs from the posterior to the anterior areas are plotted on the abscissa. The symbols represent the various task conditions (e.g., easy or difficult direction discrimination). The horizontal line indicates the point at which activations in the two groups would be equal. The areas above and below the horizontal line indicate greater or less activation in the patient group in comparison with that in the control group. The two inserts on the right show the group differences (SZ - NC) in BOLD signal changes under easy and difficult task conditions in left ICPFC (top) and MT+ (bottom), and the symbols represent each type of task (e.g., direction discrimination).

ther group. This result is not entirely surprising for several reasons. First, monkey studies have shown correlations between behavioral responses and neuronal responses in MT, but only when the task was simply detection of motion in the presence of motion (Britten, Shadlen, Newsome, \& Movshon, 1992). When a motion task required more than one choice (e.g., Which of two sequentially presented targets moves to the right?), the behavioral responses of monkeys were correlated with the responses of MT neurons only during the first, but not during the second, presentation of the motion stimulus, and the behavioral responses were not correlated with responses of PFC neurons (Zaksas \& Pasternak, 2006). Our tasks, like the one used in the Zaskas and Pasternak monkey study, required a choice over the two presentations of the visual stimulus, and our results in humans are consistent with their results in nonhuman primates. Second, the fMRI response represents averaged activation over all trials, not just to a single stimulus or to a first stimulus presentation. The absence of a significant correlation between behavioral and cortical responses suggests that other neural processes, in addition to activation of relevant cortical regions, may impact behavioral decisions during motion perception. Our sample size was relatively small, which may have obscured a significant correlation, however.

\section{Sensory Processing}

Our finding of decreased cortical activity in area MT + in schizophrenia was unexpected for two reasons. First, one previous study showed that patients with schizophrenia had a higher BOLD response than did controls in occipital regions during passive viewing of visual stimuli (Renshaw, Yurgelun-Todd, \& Cohen, 1994). Our experiment, however, required participants to engage actively in a visual discrimination task, and our results may therefore reflect a group difference that becomes apparent during active engagement of motion-specialized visual mechanisms. Second, BOLD signal levels increase linearly with luminance contrast in V1 (Boynton, Engel, Glover, \& Heeger, 1996) and with motion signal strength in MT+ (Rees et al., 2000) in normal participants. We therefore expected patients with schizophrenia to show heightened activation in posterior visual areas because the stimulus strengths of the motion stimuli had to be increased in order for them to perform with accuracy equivalent to that of the controls. However, we found the opposite: BOLD signals in motion-specialized extrastriate cortex were reduced in spite of the increased strength of the stimuli. We find it interesting that one study found reduced activation in almost all posterior brain regions involved in visual processing when patients with schizophrenia viewed checkerboard 
stimuli (Braus, Weber-Fahr, Tost, Ruf, \& Henn, 2002), suggesting that cortical processing of simple nonmotion visual information is deficient.

It is possible that inadequate input from earlier visual areas (such as V1/V2) played a role in the reduced MT+ response to visual motion in the patients. Such an interpretation would be consistent with impaired performance by patients with schizophrenia on tasks that involve early stages of visual processing (e.g., impaired velocity discrimination; Kim et al., 2006) and hypersensitivity to backward masking (Green, Nuechterlein, \& Mintz, 1994), which implicate magnocellular inputs to dorsal stream regions. In the present study, patients with schizophrenia did show a nonsignificant trend toward lower activation than did controls in the striate cortex on the direction discrimination task ( $p=.09)$, but not on the speed discrimination task. The experimental design of the present study does not permit a disturbance in input to MT to be distinguished from local effects in MT+ itself. Thus, at least as early as the motion-specialized extrastriate cortical region (but possibly earlier), patients with schizophrenia did not fully engage sensory cortical regions during visual motion processing.

Activity in area MT + can be modulated by attention (Saenz, Buracas, \& Boynton, 2002). If attention contributed to the lowered MT activations in patients, group differences would be expected to occur not only on direction and speed tasks, but also on the contrast discrimination task, inasmuch as the three visual discrimination tasks had similar attentional requirements. The fact that group differences were found only in the two motion tasks suggests that attention is an unlikely explanation for reduced MT+ activity in patients.

\section{Beyond Sensory Processing}

Given the simplicity of the visual tasks employed, anterior brain regions were not thought to be directly involved here, and indeed, activation levels in these frontal areas were low in normal controls. The combination of reduced activation in posterior regions and increased activation in anterior left ICPFC in patients suggests that nonsensory cortical areas are recruited to compensate for reduced involvement of sensory regions that should be directly involved in the processing of motion signals. This finding complements the results of studies that have found compensatory recruitment of PFC regions in patients with schizophrenia while they were engaged in a range of cognitive and motor functions (Bonner-Jackson, Haul, Csernansky, \& Barch, 2005; Heckers et al., 1998; Nagel et al., 2007). Enhanced recruitment of regions in PFC is thought to compensate for underrecruitment of extrafrontal regions (e.g., hippocampus) or of more localized regions within PFC. Our findings of the transcortical recruitment for motion processing indicate that compensatory recruitment also occurs when certain sensory functions are deficient.

Although the ICPFC does not receive direct input from motion processing areas, it does receive indirect projections from V1 through inferior temporal cortex in the nonhuman primate (Barbas, 1988). Anatomically, the ICPFC is located inferior to the dorsolateral PFC (DLPFC). The two PFC regions also differ functionally; ICPFC supports object working memory (Wilson, O'Scalaidhe, \& Goldman-Rakic, 1993) and visual categorization (Damasio, Grabowski, Tranel, Hichwa, \& Damasio, 1996), whereas DLPFC processes spatial information.

The activation we observed in ICPFC in patients with schizophrenia would be consistent with the use of cognitive strategies to buttress strained functioning of the sensory motion processing system. Motion discrimination normally depends on sensory processing of relevant information, such as speed or direction, which can be encoded with neural responses along a continuous scale of stimulus dimensions. When sensory processing resources are not adequate, strategies such as cognitive categorization may be recruited. In speed discrimination, for example, patients could categorize motion targets into two groupsone labeled "fast," the other "slow." Such a cognitive categorization strategy, although not as precise as sensory encoding along a continuous scale, would allow a speed discrimination task to be performed on a coarse scale, but may still reveal changes in cortical activation when more fine-grained discriminations are required.

The fact that ICPFC activation was increased only on the left side suggests that patients may have used a verbally mediated cognitive strategy to supplement the downward sensory processing required by the motion tasks (Stephan et al., 2003). Visual motion-related neural responses in the PFC are modulated by task demands. In healthy people, increases in the difficulty level of motion tasks are associated with increased activity in the anterior cingulate and prefrontal cortices (Rees et al., 2000). The fact that controls did not show increased activation in anterior regions suggests that they were able to perform the motion tasks by relying on sensory regions (e.g., MT) without needing to recruit PFC. The task, therefore, was not so difficult that controls needed to recruit PFC. Patients, on the other hand, under-activated motion-sensitive sensory regions (e.g., MT) and activated PFC. We interpreted this pattern as indicating that PFC activation compensated for deficient processing in sensory regions. It seems unlikely that task difficulty per se is a parsimonious explanation for this difference between patients and controls, because the design of the present study purposefully fixed task difficulty to equivalent levels on the basis of individual participants' performance. Thus, we hypothesized that the perceptual performance of the patients was related to activation of PFC.

The significantly lower level of activation in left ICPFC in normal controls suggests that they relied primarily on sensory systems to process motion stimuli.

\section{Antipsychotic Medications}

Most of the participants with schizophrenia were being treated with antipsychotic medications, and all were clinically stable. Although this combination is considered optimal for minimizing the confounding effect of unstable clinical state on cognitive performance (Buchanan et al., 2005), it leaves open the possibility that the anomalous functional activation in the patient group during the motion 
tasks was a consequence of drug treatment. Antipsychotic medications could conceivably affect functional activation differently in different brain regions. However, if such regionally specific effects of medications accounted for the findings on the motion tasks, the same anomalous activation should also have been observed during the nonmotion task. The groups did not differ in regional activation during the nonmotion task. The functional dissociation between the motion and nonmotion tasks may therefore be more parsimoniously attributed to a deficit in the processing of motion stimuli than to a medication effect that selectively targets motion tasks, but not nonmotion tasks. This deficit results in both the underrecruitment of regions that subserve visual motion processing and the compensatory recruitment of a region that subserves higher order cognitive processing.

\section{Implications for Smooth Pursuit Eye Movements}

Motion processing provides necessary sensory signals for generating smooth pursuit eye movements (Keller \& Heinen, 1991; Newsome \& Pare, 1988; Wurtz et al., 1990). Studies of cortical responses during smooth pursuit eye movement in schizophrenia have shown mixed results. Activity in posterior regions, including MT+ or V5, was reported to be either reduced to various degrees (Lencer, Nagel, Sprenger, Heide, \& Binkofski, 2005; Tregellas et al., 2004) or somewhat increased (Hong et al., 2005). Smooth pursuit eye movements consist of initial and maintenance stages, which rely on sensory motion and extraretinal signals, respectively. The reduced activity of MT + in patients' responses to visual motion signals may be related to the initial phase of smooth pursuit (e.g., acceleration). Indeed, MT activity has been shown to be correlated with smooth pursuit eye velocity in healthy people, and this correlation was not present in schizophrenia (Lencer et al., 2005). This conjecture would be consistent with the psychophysical finding that the motion perception deficit in schizophrenia is associated with the initial, but not the maintenance, stage of smooth pursuit dysfunction (Chen, Levy, et al., 1999).

\section{Functional Processes Across \\ Different Cortical Systems}

We propose that nonmotion processing strategies that rely on prefrontal areas - specifically the left ICPFC - are recruited in schizophrenia to compensate for compromised sensory functioning. This functional shift to left ICPFC activation implicates a prefrontal area that is underinhibited in schizophrenia. The GABAergic inhibitory neurotransmission system is implicated in both schizophrenia (Benes, 2000) and visual motion processing (Egelhaaf, Borst, \& Pilz, 1990). Whether a dysfunction in the GABA system is related to the functional shift from posterior regions to the left ICPFC cannot be determined from this study. One possible consequence of recruiting a nonsensory cortical area in order to process sensory motion input is suboptimal neural processing of motion signals. Recruiting the anterior cortical system for sensory processing may also leave fewer neural resources available for cognitive processing.
The existence of a strategy of recruiting alternative pathways, although speculative, is consistent with the results of a behavioral study by Chen, Nakayama, et al. (1999). In that study, we found deficient speed discrimination in patients with schizophrenia when they judged speeds in intermediate ranges (e.g., $10 \mathrm{deg} / \mathrm{sec}$ ), where speed cues were dominant. Speed discrimination of patients was generally equivalent to that of control participants at slow speeds, such as $3 \mathrm{deg} / \mathrm{sec}$, and at fast speeds, such as $26 \mathrm{deg} / \mathrm{sec}$, because they substituted nonmotion position or contrast cues in order to process motion information. Our imaging data raise the possibility that an analogous substitution strategy may be mirrored in the patients' altered neural activation patterns.

The altered response pattern of patients with schizophrenia during motion processing tasks occurs across the posterior and anterior cortical areas, which are putatively designated for sensory and cognitive functions, respectively. We observed group differences with large effect sizes in both MT+ and left ICPFC, even in these relatively modest samples, suggesting that neural activity in schizophrenia is not simply dampened in the visual system, but is more systematically altered across sensory and cognitive domains. This altered neural activity across a cortical network is associated specifically with motion perception, a type of visual behavior that requires dynamic integration of both spatial and temporal information (Nakayama, 1985).

We did not find a significant correlation between peak activations of the MT and the PFC in either group. However, our sample size may have been too small to identify a significant inverse relationship between activation in these two regions. A significant correlation would be consistent with the existence of time-locked compensatory activities between different cortical areas, but the causal relationships are still not completely determined. It should be noted that the present study was not designed to test for functional connectivity. A rigorous test of the connectivity hypothesis would require faster event dynamics and more frequent sampling (our TR was set for $3 \mathrm{sec}$ for maximal activations). The differential cortical activations during the motion perception tasks, found in this study, suggest that future studies of functional connectivity between different cortical areas in the context of the processing of motion signals are warranted.

Although the exact manner in which neural activities are altered in schizophrenia needs to be specified in further detail, motion processing may tap into vulnerabilities created by alterations in neural organization. Nevertheless, the engagement of multiple cortical systems during "low level" visual motion tasks in schizophrenia presents evidence that the cortex is remarkably adaptable in modifying its functional specificity, even across the domains of sensory and cognitive processing.

\section{AUTHOR NOTE}

We dedicate this article to the memory of P.S.H., whose vision and support inspired this study. The authors thank Francine Benes, Steven Matthysse, and Charles Stromeyer for helpful comments on previous drafts of this article, and Daniel Norton for technical help. This work was 
supported in part by grants from NIMH, NARSAD, the Milton Fund of Harvard University, the Hunt Foundation, the Essel Foundation, and the Sidney R. Baer, Jr. Foundation. Correspondence concerning this article should be addressed to Y. Chen, McLean Hospital, Department of Psychiatry, Harvard Medical School, RM G06, Centre Bldg., 115 Mill St., Belmont, MA 02478 (e-mail: ychen@mclean.harvard.edu).

\section{REFERENCES}

American Psychiatric Association (1994). Diagnostic and statistical manual of mental disorders (4th ed.). Washington, DC: Author.

ANDREASEN, N. C. (1999). A unitary model of schizophrenia: Bleuler's "fragmented phrene" as schizencephaly. Archives of General Psychiatry, 56, 781-787.

BARBAS, H. (1988). Anatomic organization of basoventral and mediodorsal visual recipient prefrontal regions in the rhesus monkey. Journal of Comparative Neurology, 276, 313-342.

BENEs, F. M. (2000). Emerging principles of altered neural circuitry in schizophrenia. Brain Research Reviews, 31, 251-269.

Bleuler, E. (1950). Dementia praecox or the group of schizophrenias (J. Zinkin, Trans.). New York: International Universities Press. (Original work published 1911)

Bonner-Jackson, A., Haul, K., Csernansky, J. G., \& Barch, D. M. (2005). The influence of encoding strategy on episodic memory and cortical activity in schizophrenia. Biological Psychiatry, 58, 47-55.

Boynton, G. M., Engel, S. A., Glover, G. H., \& Heeger, D. J. (1996). Linear systems analysis of functional magnetic imaging in human V1. Journal of Neuroscience, 16, 4207-4221.

Braus, D. F., Weber-Fahr, W., Tost, H., Ruf, M., \& Henn, F. A. (2002). Sensory information processing in neuroleptic-naive firstepisode schizophrenic patients: A functional magnetic resonance imaging study. Archives of General Psychiatry, 59, 696-701.

Britten, K. H., Shadlen, M. N., Newsome, W. T., \& Movshon, J. A. (1992). The analysis of visual motion: A comparison of neuronal and psychophysical performance. Journal of Neuroscience, 12, 4745-4765.

Brodmann, K. (1909). Vergleichende Lokalisationslehre der Grosshirnrinde in ihren Prinzipien dargestellt auf Grund des Zellenbaues. Leipzig: Johann Ambrosius Barth Verlag.

Buchanan, R. W., Davis, M., Goff, D., Green, M. F., Keefe, R. S. E., LEON, A. C., ET AL. (2005). A summary of the FDA-NIMH-MATRICS workshop on clinical trial design for neurocognitive drugs for schizophrenia. Schizophrenia Bulletin, 31, 5-19.

Chen, Y., Levy, D. L., Nakayama, K., Matthysse, S., Palafox, G., \& Holzman, P. S. (1999). Dependence of impaired eye tracking on deficient velocity discrimination in schizophrenia. Archives of General Psychiatry, 56, 155-161.

Chen, Y., Nakayama, K., Levy, D. L., Matthysse, S., \& Holzman, P. S. (1999). Psychophysical isolation of a motion-processing deficit in schizophrenics and their relatives and its association with impaired smooth pursuit. Proceedings of the National Academy of Sciences, 96, 4724-4729.

Chen, Y., Nakayama, K., Levy, D. L., Matthysse, S., \& Holzman, P. S. (2003). Processing of global, but not local, motion direction is deficient in schizophrenia. Schizophrenia Research, 61, 215-227.

Coyle, J. T. (1996). The glutamatergic dysfunction hypothesis for schizophrenia. Harvard Review of Psychiatry, 5, 241-253.

Damasio, H., Grabowski, T. J., Tranel, D., Hichwa, R. D., \& Damasio, A. R. (1996). A neural basis for lexical retrieval. Nature, 380, 499-505.

Edelman, G. M. (2003). Naturalizing consciousness: A theoretical framework. Proceedings of the National Academy of Sciences, $\mathbf{1 0 0}$, $5520-5524$

EgelhaAf, M., Borst, A., \& Pilz, B. (1990). The role of GABA in detecting visual motion. Brain Research, 509, 156-160.

Friston, K. J., Worsley, K. J., Frackowiak, R. S. J., Mazziotta, J. C., \& Evans, A. C. (1993). Assessing the significance of focal activations using their spatial extent. Human Brain Mapping, 1, 210-220.

GoldMan-RaKic, P. S. (1988). Topography of cognition: Parallel distributed networks in primate association cortex. Annual Review of Neuroscience, 11, 137-156.

Green, M. F., Nuechterlein, K. H., \& Mintz, J. (1994). Backward masking in schizophrenia and mania: I. Specifying a mechanism. $A r-$ chives of General Psychiatry, 51, 939-944.

Greicius, M. D., \& Menon, V. (2004). Default-mode activity during a passive sensory task: Uncoupled from deactivation but impacting activation. Journal of Cognitive Neuroscience, 16, 1484-1492.

Heckers, S., Rauch, S. L., Goff, D., Savage, C. R., Schacter, D. L., Fischman, A. J., \& Alpert, N. M. (1998). Impaired recruitment of the hippocampus during conscious recollection in schizophrenia. $\mathrm{Na}$ ture Neuroscience, 1, 318-323.

Hollingshead, A. B. (1957). Two factor index of social position. New Haven, CT: Author.

Holzman, P. S., Proctor, L. R., \& Hughes, D. W. (1973). Eye-tracking patterns in schizophrenia. Science, 181, 179-181.

Hong, L. E., Tagamets, M., Avila, M., Wonodi, I., Holcomb, H., \& Thaker, G. K. (2005). Specific motion processing pathway deficit during eye tracking in schizophrenia: A performance-matched functional magnetic resonance imaging study. Biological Psychiatry, 57, 726-732.

Huk, A. C., \& HeEger, D. J. (2002). Pattern-motion responses in human visual cortex. Nature Neuroscience, 5, 72-75.

Keller, E. L., \& Heinen, S. J. (1991). Generation of smooth-pursuit eye movements: Neuronal mechanisms and pathways. Neuroscience Research, 11, 79-107.

Kendler, K. S., Leiberman, J. A., \& Walsh, D. (1989). Structured interview for schizotypy (SIS): A preliminary report. Schizophrenia Bulletin, 15, 559-571.

Kim, D., Wylie, G., Pasternak, R., Butler, P. D., \& Javitt, D. C. (2006). Magnocellular contributions to impaired motion processing in schizophrenia. Schizophrenia Research, 82, 1-8.

Kraepelin, E. (1919). Dementia praecox and paraphrenia. Chicago: Medical Books.

Lencer, R., Nagel, M., Sprenger, A., Heide, W., \& Binkofski, F. (2005). Reduced neuronal activity in the V5 complex underlies smooth-pursuit deficit in schizophrenia: Evidence from an fMRI study. NeuroImage, 24, 1256-1259.

Levin, S., Luebke, A., Zee, D. S., Hain, T. C., Robinson, D. A., \& Holzman, P. S. (1988). Smooth pursuit eye movements in schizophrenics: Quantitative measurements with the search-coil technique. Journal of Psychiatric Research, 22, 195-206.

Levy, D. L., Holzman, P. S., Matthysse, S., \& Mendell, N. R. (1993). Eye tracking dysfunction and schizophrenia: A critical perspective. Schizophrenia Bulletin, 19, 461-536.

Nagel, M., Sprenger, A., NitschKe, M., Zapf, S., Heide, W., BinKofSKI, F., \& LENCER, R. (2007). Different extraretinal neuronal mechanisms of smooth pursuit eye movements in schizophrenia: An fMRI study. Neurolmage, 34, 300-309.

NAKAYAMA, K. (1985). Biological image motion processing: A review. Vision Research, 25, 625-660.

Newsome, W. T., \& Pare, E. B. (1988). A selective impairment of motion perception following lesions of the middle temporal visual area (MT). Journal of Neuroscience, , 8, 2201-2211.

PARK, S., \& Holzman, P. S. (1992). Schizophrenics show spatial working memory deficits. Archives of General Psychiatry, 49, 975-982.

Plant, G. T., \& Nakayama, K. (1993). The characteristics of residual motion perception in the hemifield contralateral to lateral occipital lesions in humans. Brain, 116, 1337-1353.

ReEs, G., Friston, K., \& KoCH, C. (2000). A direct quantitative relationship between the functional properties of human and macaque V5. Nature Neuroscience, 3, 716-723.

Renshaw, P. F., Yurgelun-Todd, D. A., \& Cohen, B. M. (1994). Greater hemodynamic response to photic stimulation in schizophrenic patients: An echo planar MRI study. American Journal of Psychiatry, 151, 1493-1495.

Saenz, M., Buracas, G. T., \& Boynton, G. M. (2002). Global effects of feature-based attention in human visual cortex. Nature Neuroscience, 5, 631-632.

Spitzer, R., Williams, J., Gibbon, M., \& First, M. (1994). Structured clinical interview for DSM-IV (Patient ed.). Washington, DC: American Psychiatric Association.

Stephan, K. E., Marshall, J. C., Friston, K. J., Rowe, J. B., Ritzl, A., ZILLES, K., \& FINK, G. R. (2003). Lateralized cognitive processes and lateralized task control in the human brain. Science, 301, 384-386. 
Stuve, T. A., Friedman, L., Jesberger, J. A., Gilmore, G. C., Strauss, M. E., \& Meltzer, H. (1997). The relationship between smooth pursuit performance, motion perception and sustained visual attention in patients with schizophrenia and normal controls. Psychological Medicine, 27, 143-152.

Sweeney, J. A., Clementz, B. A., Haas, G. L., Escobar, M. D., DraKe, K., \& Frances, A. J. (1994). Eye tracking dysfunction in schizophrenia: Characterization of component eye movement abnormalities, diagnostic specificity, and the role of attention. Journal of Abnormal Psychology, 103, 222-230.

TAlairach, J., \& Tournoux, P. (1988). Co-planar stereotaxic atlas of the human brain: 3-Dimensional proportional system-An approach to cerebral imaging. New York: Thieme.

Thaker, G. K., Ross, D. E., Cassady, S. L., Adami, H. M., LaPorte, D., Medoff, D. R., \& Lahti, A. (1998). Smooth pursuit eye movements to extraretinal motion signals: Deficits in relatives of patients with schizophrenia. Archives of General Psychiatry, 55, 830-836.

Tootell, R. B. H., Reppas, J. B., Kwong, K. K., Malach, R., BORN, R. T., BRADY, T. J., ET AL. (1995). Functional analysis of human MT and related visual cortical areas using magnetic resonance imaging. Journal of Neuroscience, 15, 3215-3230.

Tregellas, J. R., Tanabe, J. L., Miller, D. E., Ross, R. G., Olincy, A.,
\& Freedman, R. (2004). Neurobiology of smooth pursuit eye movement deficits in schizophrenia: An fMRI study. American Journal of Psychiatry, 161, 315-321.

VAn Essen, D. C., \& MAunsell, J. H. (1983). Hierarchical organization and functional streams in the visual cortex. Trends in Neurosciences, 6, 370-375.

Wilson, F. A., O’Scalaidhe, S. P., \& Goldman-Rakic, P. S. (1993). Dissociation of object and spatial processing domains in primate prefrontal cortex. Science, 260, 1955-1958.

Woods, S. W. (2003). Chlorpromazine equivalent doses for the newer atypical antipsychotics. Journal of Clinical Psychiatry, 64, 663-667.

Wurtz, R. H., Yamasaki, D. S., Duffy, C. J., \& RoY, J.-P. (1990). Functional specialization for visual motion processing in primate cerebral cortex. Cold Spring Harbor Symposia on Quantum Biology, 55, 717-727.

ZAKSAS, D., \& PASTERnaK, T. (2006). Directional signals in the prefrontal cortex and in area MT during working memory for visual motion task. Journal of Neuroscience, 26, 11726-11742.

(Manuscript received June 6, 2007; revision accepted for publication January 29, 2008.) 